\title{
The different reasons in the first medical examination related to the severity and the subsequent treatment of type 2 diabetes
}

\author{
Hideyuki Motohashi ${ }^{1,2}$, Naoka Wada $^{1}$, Haruka Ota ${ }^{1}$, Yoshitaka Yano ${ }^{1}$, Makoto Miura ${ }^{2}$ \\ ${ }^{I}$ Education and Research Center for Clinical Pharmacy, Kyoto Pharmaceutical University, Japan, ${ }^{2}$ Department of \\ Pharmacy, Rakuwakai Otowa Hospital, Japan
}

Background: An increased number of diabetic patients is a serious problem and the total number of diabetic patients is about 3.16 million in Japan. Although specific health checkups and health guidance started from 2008, the implementation rate is $48.6 \%$ in 2014 , which did not reach at $70 \%$ that is the aim of Ministry of Health, Labour and Welfare. Thus, early detection of diabetes should be facilitated. In this study, we investigated whether the different reasons for the first medical examination were related to the severity of the type 2 diabetes and the subsequent treatment options.

Methods: This study was a retrospective surveillance of diabetic patients in Rakuwakai Otowa hospital, Kyoto, Japan. Patients were diagnosed as type 2 diabetes for the first time from June, 2014 to December, 2014. Patients were divided to three groups, Detected by health examination group(I), Detected by other reasons group (II) and Leave untreated group (III). Age, gender, BMI, HbA1c, blood-glucose-level, prescribed drugs were surveyed. Age, HbA1c, and BG among groups were compared by Tukey's test and number of prescribed drugs among groups was compared by the Chi-squared test. This study was approved by ethics committees of Kyoto Pharmaceutical University and Rakuwakai Otowa Hospital.

Results: In Detected by health examination group (I), HbA1c level and blood-glucose level were significantly lower than those of Detected by other reasons (II) and of Leave untreated groups (III) $(\mathrm{p}<0.05)$. It suggested that health examination contributed to early detection of type 2 diabetes. In addition, in Detected by health examination group (I), fewer patients were prescribed comparing with other two groups (II, III) $(p<0.05)$. It is suggested that the difference of the reasons in the first consultation are related to the subsequent treatment options for type 2 diabetes.

Conclusion: Medical examination plays an important role in early detection and treatment in diabetic patients in this study. In addition, we should make more efforts to prevent progression of type 2 diabetes. 